06.5:08.2

\title{
Особенности роста слоев в напряженных сверхрешетках InAs/GaSb
}

\author{
(ㄱ Р.В. Левин, В.Н. Неведомский, Л.А. Сокура \\ Физико-технический институт им. А.Ф. Иоффе РАН, Санкт-Петербург, Россия \\ E-mail: Lev@vpegroup.ioffe.ru
}

Поступило в Редакцию 20 июля 2021 г.

В окончательной редакции 4 октября 2021 г.

Принято к публикации 18 октября 2021 г.

\begin{abstract}
Представлены результаты исследования факторов, влияющих на толщину переходных слоев в напряженных сверхрешетках InAs/GaSb при их выращивании методом газофазной эпитаксии из металлоорганических соединений. Показано, что толщины интерфейсных слоев между InAs и GaSb практически не зависят от температуры роста. Регистрируемое влияние на толщину интерфейсных слоев оказывает направление переключения роста слоев. Наименьшая толщина $1.2-1.4 \mathrm{~nm}$ интерфейсного слоя была получена для направления переключения роста c GaSb на InAs.
\end{abstract}

Ключевые слова: газофазная эпитаксия из металлоорганических соединений, напряженная сверхрешетка, $\mathrm{InAs} / \mathrm{GaSb}$, интерфейсный слой, просвечивающая электронная микроскопия.

DOI: 10.21883/PJTF.2022.03.51974.18966

Фотоприемники, работающие в ближнем и среднем инфракрасном (ИК) диапазоне, могут использоваться в самых различных областях: от экологии (для детектирования вредных веществ в атмосфере) до военного применения. Разработка и создание таких устройств представляют собой весьма перспективную задачу. В настоящее время основной системой материалов для ИКфотоприемников является $\mathrm{HgCdTe}$. Напряженные сверхрешетки на основе InAs/GaSb позволяют расширить класс оптоэлектронных приборов для среднего и ближнего ИК-диапазона (вплоть до $15 \mu \mathrm{m}$ ), их использование является альтернативным подходом, который позволит понизить скорость оже-рекомбинации и, как следствие, повысить рабочую температуру $[1,2]$.

Основным методом изготовления сверхрешеток InAs/GaSb в настоящее время является метод молекулярно-пучковой эпитаксии (МПЭ) [3-5]. Как следует из литературных данных [6], минимальная толщина интерфейсных (т.е. переходных) слоев $\mathrm{InAs} / \mathrm{GaSb}$ для метода МПЭ составляет около 1 монослоя $(0.3 \mathrm{~nm})$. Однако для массового производства приборов более предпочтительной технологией получения является газофазная эпитаксия из металлоорганических соединений (ГФЭМОС) по причине более низкой себестоимости. В настоящее время ГФЭМОС приоритетно используется для получения толстых (более $3 \mu \mathrm{m}$ ) слоев $\mathrm{A}^{3} \mathrm{~B}^{5}$, в частности InAs и GaSb. Однако при выращивании тонкослойных сверхрешеток возрастают требования к резкости и качеству интерфейса между слоями. Поэтому для метода ГФЭМОС необходимо проведение комплекса исследований стадий роста, которые влияют на резкость гетерограниц и на „качество“ квантовых ям, от которых зависят рабочие характеристики гетероструктуры. Например, в работе [7] была изучена зависимость толщины интерфейсных слоев в сверхрешетках InAs/GaSb, получаемых методом ГФЭМОС, от направления переключе- ния роста c InAs на GaSb и наоборот. Их минимальная толщина составила $2.5-3.8$ монослоев $(0.75-1.14 \mathrm{~nm})$.

Целью настоящей работы является изучение влияния температуры, условий и динамики переключения роста слоев $\mathrm{InAs} / \mathrm{GaSb} / \mathrm{InAs}$ на резкость и качество гетерограниц.

Структуры выращивались на установке ГФЭМОС AIX-200 (AIXTRON, Германия) на подложках $n$-GaSb (001). Давление в реакторе составляло $100 \mathrm{mbar}$. Газноситель - очищенный водород с точкой росы не хуже $-100^{\circ} \mathrm{C}$, суммарный поток через реактор составлял $5.5 \mathrm{slpm}$. Источниками элементов для роста были следующие соединения: триметилиндий (TMIn), триэтилгаллий (TEGa), триметилсурьма (TMSb) и арсин $\left(\mathrm{AsH}_{3}\right)$. Соотношение элементов V/III (условие полного пиролитического разложения реагентов) составило 93 для InAs и 22.5 для $\mathrm{GaSb}$. Скорости роста InAs и $\mathrm{GaSb}$ поддерживались на одном уровне.

Полученные структуры были исследованы методом просвечивающей электронной микроскопии (ПЭМ) на микроскопе JEM-2100F (Jeol, Япония) при ускоряющем напряжении $200 \mathrm{kV}$ в режиме дифракционного контраста (двулучевые условия). Подготовка проб осуществлялась стандартным для гетероструктур методом: шлифовка с последовательным уменьшением размера зерна абразива и распылением ионами $\mathrm{Ar}^{+}$с энергией $4-0.5 \mathrm{keV}$ под скользящими углами на конечном этапе.

Измерение толщин слоев проводилось по темнопольным ПЭМ-изображениям, полученным в двулучевых условиях с действующим вектором дифракции $\mathbf{g}=(002)$. В этом режиме механизм формирования контраста такой, что на изображении слои $\mathrm{GaSb}$ отделены от слоев InAs тонкой полосой темного контраста, которая соответствует переходному слою $\mathrm{In}(\mathrm{GaAs}) \mathrm{Sb}$ с постепенным взаимозамещением компонентов в подрешетках III и V групп. Границы слоев InAs/GaSb определялись по мини- 
мумам контраста на профиле интенсивности, который соответствует середине переходного слоя $\mathrm{In}(\mathrm{GaAs}) \mathrm{Sb}$. Границы же самого переходного слоя $\mathrm{In}(\mathrm{GaAs}) \mathrm{Sb}$ определялись на половинах высоты перепада интенсивности слоев $\operatorname{In}(\mathrm{GaAs}) \mathrm{Sb} / \mathrm{InAs}$ и $\mathrm{In}(\mathrm{GaAs}) \mathrm{Sb} / \mathrm{GaSb}$.

Также следует заметить, что разрешающая способность ПЭМ в дифракционном режиме хуже, чем в режиме разрешения решетки, из-за меньшей апертуры объектной диафрагмы. Однако при используемой диафрагме пространственное разрешение ПЭМ будет не хуже $0.6 \mathrm{~nm}$.

Проведенные исследования условно можно разделить на выявление двух закономерностей:

1) влияния температуры роста на толщину переходных слоев $\mathrm{In}(\mathrm{GaAs}) \mathrm{Sb}$ при прочих равных условиях;

2) влияния условий переключения подачи газов на толщину переходных слоев $\mathrm{In}(\mathrm{GaAs}) \mathrm{Sb}$ при выбранной температуре роста.

Для исследования влияния температуры роста на толщину интерфейсных слоев была выращена структура, состоящая из четырех групп по два слоя InAs в GaSb. Каждая группа выращивалась при постоянной температуре в диапазоне от 480 до $540^{\circ} \mathrm{C}$ с шагом $20^{\circ} \mathrm{C}$. Толщина GaSb между группами была задана $30 \mathrm{~nm}$, между слоями InAs внутри группы и толщина самих слоев InAs - $15 \mathrm{~nm}$. Потоки реагентов элементов V и III групп поддерживались постоянными, а изменялась только температура роста с необходимой выдержкой для стабилизации ее возможных колебаний.

Конечно, при таком дизайне структуры возможные протяженные дефекты и другие особенности нижних слоев будут влиять на рост вышележащих слоев. Однако мы предположили (и это подтвердилось результатами ПЭМ-исследований), что нижние группы слоев будут иметь небольшую плотность прорастающих протяженных дефектов. А дефекты, генерирующиеся на конкретном слое, можно определять дифференциальным методом.

На изображении поперечного сечения структуры (рис. $1, a$ ) в режиме, чувствительном к изменениям химического состава (темное поле с действующим дифракционным вектором $\mathbf{g}=(002))$, хорошо видны четкие границы слоев InAs и GaSb, что удобно для определения их толщины. Протяженные дефекты можно наблюдать в других дифракционных условиях, например с действующим дифракционным вектором $\mathbf{g}=(220)$ (рис. $1, b)$.

Помимо толщин слоев InAs, GaSb и переходных слоев $\operatorname{In}(\mathrm{GaAs}) \mathrm{Sb}$ по ПЭМ-изображениям было оценено качество структуры, а также наличие протяженных дефектов. Из рис. 1 видно, что в первой (нижней) паре слоев $\mathrm{InAs} / \mathrm{GaSb}$ (температура роста $480^{\circ} \mathrm{C}$ ) можно наблюдать неоднородность толщины слоев в латеральном направлении, промежуток между слоями InAs очень тонкий, встречаются дефекты-включения, создающие локальные упругие напряжения полусферической формы. Для второй пары (температура роста $500^{\circ} \mathrm{C}$ ) наблюдается улучшение однородности толщин слоев,
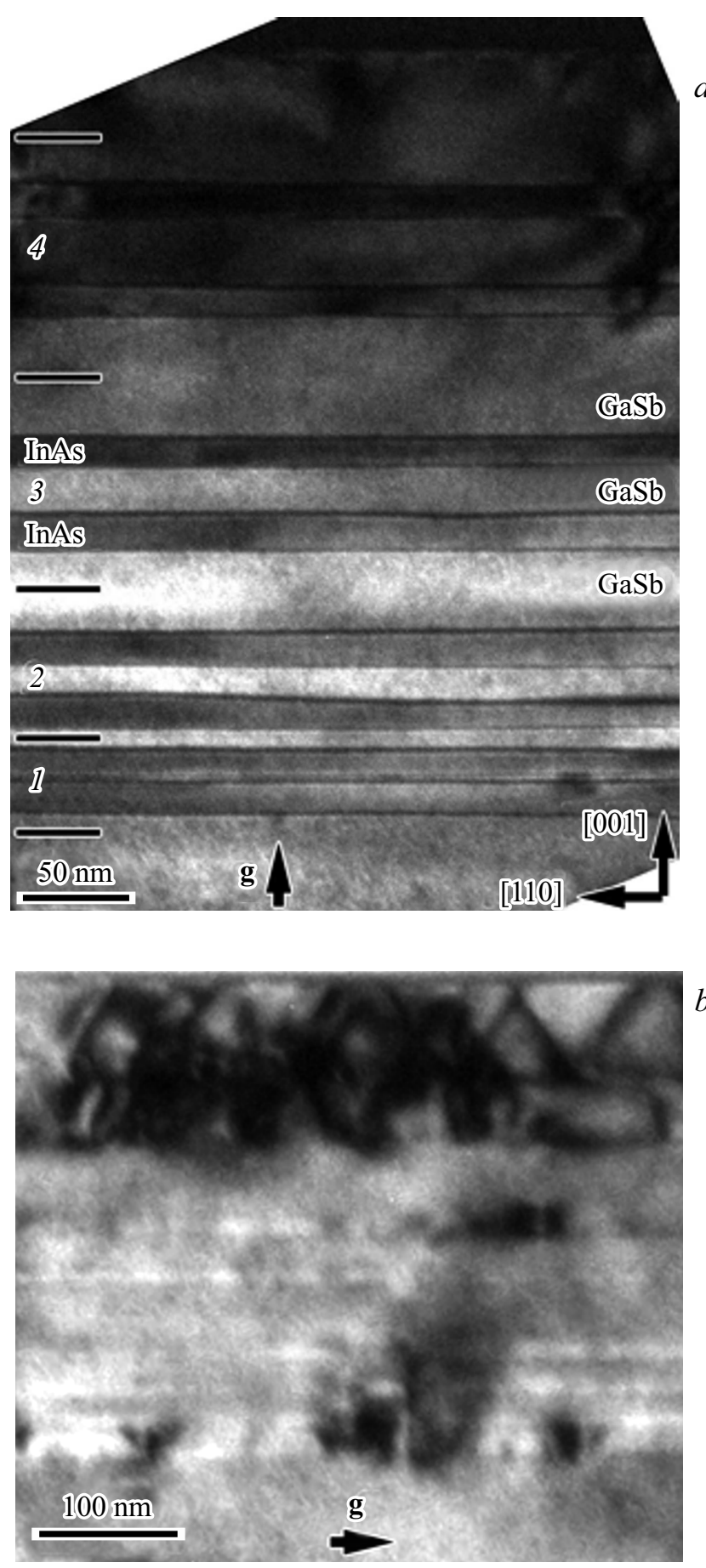

Рис. 1. ПЭМ-изображение поперечного сечения (1̄0) образца со слоями $\mathrm{InAs} / \mathrm{GaSb}$, полученными при температурах $480-540^{\circ} \mathrm{C}$, в различных дифракционных условиях. $a-$ темнопольное изображение с действующим дифракционным вектором $\mathbf{g}=(002)$. Цифрами $1-4$ указаны номера групп слоев, горизонтальные отрезки между цифрами - условное разделение между группами из двух пар слоев $\mathrm{InAs} / \mathrm{GaSb}$. $b$ - светлопольное изображение с действующим дифракционным вектором $\mathbf{g}=(220)$. 

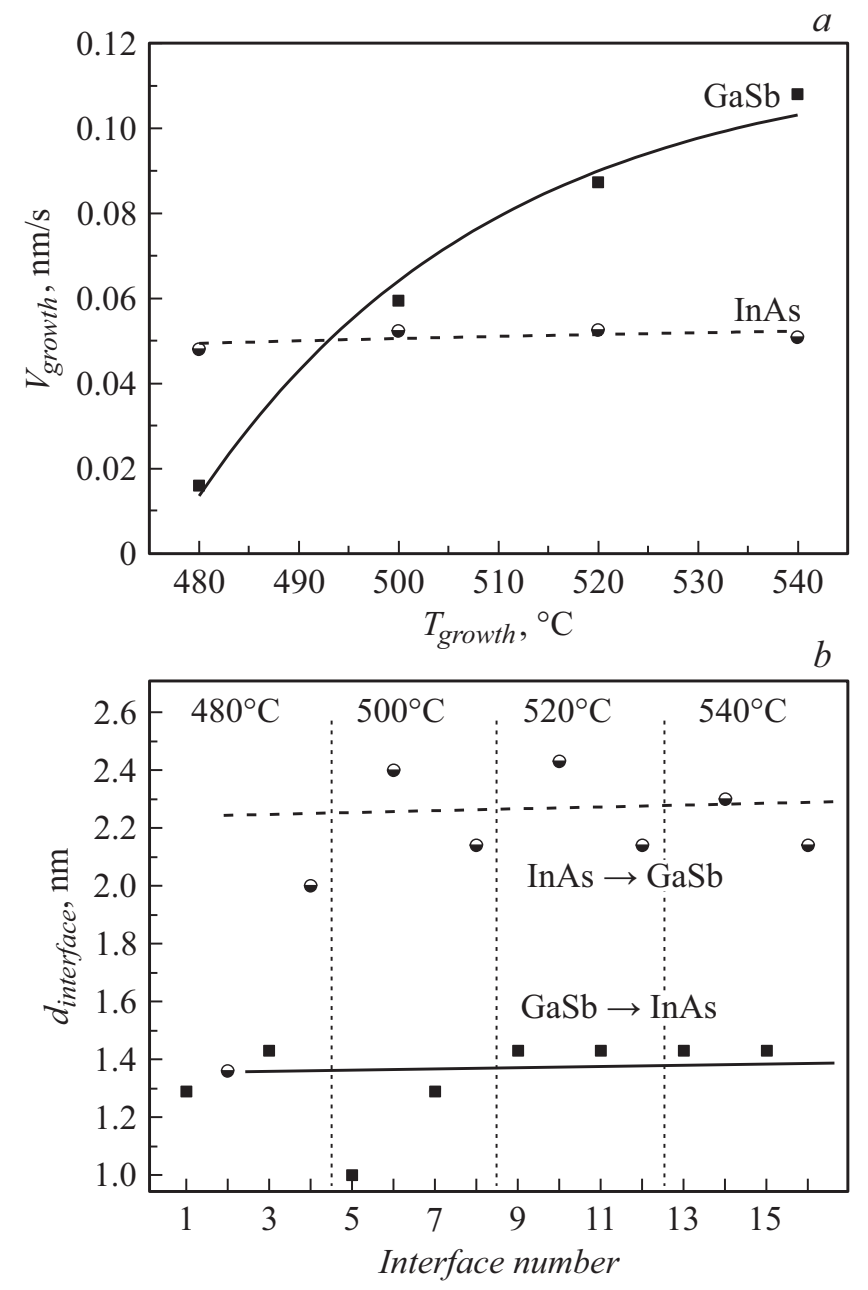

Рис. 2. Зависимости скорости роста бинарных слоев InAs и $\mathrm{GaSb}$ от температуры роста $(a)$ и толщины переходного слоя между InAs/GaSb от его номера и соответственно температуры роста $(b)$.

меньшее количество дефектов. Для третьей пары слоев (температура роста $520^{\circ} \mathrm{C}$ ) обнаружена лучшая в этом образце однородность толщины слоев, однако встречается некоторое количество протяженных дефектов. Для четвертой (самой верхней) пары слоев (температура роста $540^{\circ} \mathrm{C}$ ) однородность толщины в латеральном направлении такая же, как и в третьей паре, однако плотность протяженных дефектов на один-два порядка больше, чем во всех нижележащих слоях. Из рис. $1, b$ очевидно, что дефектов больше всего именно в четвертой паре. На основании изложенного выше был сделан вывод, что оптимальная температура роста на установке AIX-200 составляет $500-520^{\circ} \mathrm{C}$, в этом случае в слоях генерируется минимальное количество дефектов и сами слои имеют приемлемую однородность толщины. Поэтому для выявления закономерности влияния условий переключения подачи газов на толщину переходных слоев $\operatorname{In}(\mathrm{GaAs}) \mathrm{Sb}$ при фиксированной температуре роста была выбрана температура $500^{\circ} \mathrm{C}$.
Помимо оценки плотности дефектов по результатам ПЭМ-исследований были проведены измерения толщины отдельных слоев InAs, GaSb и толщины интерфейсных слоев $\operatorname{In}(\mathrm{GaAs}) \mathrm{Sb}$ между InAs/GaSb (рис. 2, b). Скорости роста слоев InAs, GaSb (рис. 2, a) были оценены по толщине слоев.

Из рис. 2, $a$ видно, что скорость роста слоев InAs в диапазоне температур $480-540^{\circ} \mathrm{C}$ остается постоянной. Однако скорость роста слоев для $\mathrm{GaSb}$ зависит от температуры роста и с увеличением последней возрастает в 6 раз. Такое поведение скорости роста, вероятно, связано с увеличением эффективности пиролитического разложения $\mathrm{TMSb}$ с $13 \%$ при $480^{\circ} \mathrm{C}$ до $90 \%$ при $540^{\circ} \mathrm{C}[8]$, что в свою очередь приводит к увеличению соотношения TMSb/TEGa с 2.9 до 20.3.

Из зависимости, представленной на рис. 2, $b$, можно видеть, что толщины интерфейсных слоев между InAs/GaSb от температуры роста практически не зависят. И при синхронной подаче обоих реагентов на толщину интерфейсных слоев оказывает влияние направление переключения роста слоев. Меньшая толщина интерфейсного слоя между InAs/GaSb в данном эксперименте была получена для направления переключения роста с $\mathrm{GaSb}$ на InAs и составила $1.2-1.4 \mathrm{~nm}$, что близко к значению толщины интерфейсного слоя, полученному в работе [7]. Возможно, образование более толстых интерфейсных слоев $\operatorname{In}(\mathrm{GaAs}) \mathrm{Sb}$ при направлении переключения роста $\mathrm{InAs} \rightarrow \mathrm{GaSb}$ связано с повышенной скоростью роста бинарных слоев $\mathrm{GaSb}$.

Для выявления закономерностей влияния условий переключения подачи газов на толщину переходных слоев $\mathrm{In}(\mathrm{GaAs}) \mathrm{Sb}$ была выращена структура аналогичного дизайна (четыре группы по два слоя InAs в $\mathrm{GaSb}$ ) при температуре роста $500^{\circ} \mathrm{C}$. При росте разных групп слоев использовались различные задержки при переключении реагентов. Так же как и выше, измерялись толщины переходных слоев $\operatorname{In}(\mathrm{GaAs}) \mathrm{Sb}$, оценивалась однородность толщины слоев InAs и $\mathrm{GaSb}$, учитывалась плотность возникающих протяженных дефектов.

На рис. 3 построена зависимость толщины интерфейсного слоя от последовательности подачи реагентов и направления переключения роста. Светлыми квадратами отмечена последовательность $\mathrm{GaSb} \rightarrow \mathrm{InAs:}$ после роста $\mathrm{GaSb}$ подавался стабилизационный поток TMSb в течение $2-10 \mathrm{~s}$, далее следовала продувка водородом в течение $30 \mathrm{~s}$, а затем рост слоя InAs начинали с введения в реактор $\mathrm{AsH}_{3}$ в течение 2-10s с последующей подачей TMIn. Темными квадратами отмечена последовательность InAs $\rightarrow \mathrm{GaSb}$ : после роста InAs подавался стабилизационный поток $\mathrm{AsH}_{3}$ в течение 2-10 s, далее следовала продувка водородом в течение $30 \mathrm{~s}$, а затем рост слоя $\mathrm{GaSb}$ начинали с введения в реактор $\mathrm{TMSb}$ на 2-10 s раньше TEGa.

Минимальная толщина интерфейсного слоя наблюдалась для направления переключения роста $\mathrm{GaSb} \rightarrow \mathrm{InAs}$ и подачи $\mathrm{AsH}_{3}$ на $2 \mathrm{~s}$ раньше TMIn. Как видно из рис. 3 , увеличение времени упреждения подачи элемента 


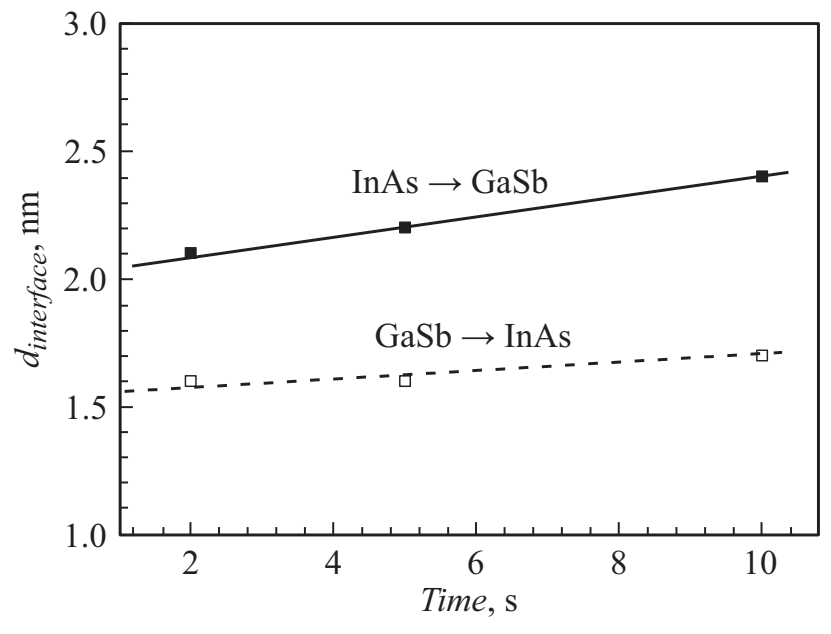

Рис. 3. Зависимость толщины интерфейсного слоя от направления переключения роста и времени упреждения подачи реагентов относительно друг друга.

$\mathrm{V}$ группы в обеих последовательностях приводило к увеличению интерфейсного слоя. Следует отметить, что направление переключения роста InAs $\rightarrow \mathrm{GaSb}$ более чувствительно ко времени упреждения подачи элемента V группы относительно элемента III группы, что выражается в увеличении толщины интерфейсного слоя с $2.1 \mathrm{~nm}$ при упреждении на $2 \mathrm{~s}$ до $2.4 \mathrm{~nm}$ при упреждении на $10 \mathrm{~s}$.

Проведенные исследования показали, что толщина интерфейсных слоев $\operatorname{In}(\mathrm{GaAs}) \mathrm{Sb}$ на границе бинарных слоев InAs/GaSb практически не зависит от температуры роста. Тем не менее оптимальная температура роста на установке AIX-200 находится в диапазоне $500-520^{\circ} \mathrm{C}$, так как при такой температуре в слоях генерируется наименьшая плотность протяженных дефектов. Основное влияние на толщину интерфейсных слоев $\operatorname{In}(\mathrm{GaAs}) \mathrm{Sb}$ оказывает направление переключения газов-источников реагентов. Наименьшая толщина $1.2-1.4 \mathrm{~nm}$ была зарегистрирована для направления переключения роста с $\mathrm{GaSb}$ на InAs при подаче $\mathrm{AsH}_{3}$ всего на $2 \mathrm{~s}$ раньше TMIn.

\section{Конфликт интересов}

Авторы заявляют, что у них нет конфликта интересов.

\section{Список литературы}

[1] C.H. Grein, P.M. Young, H. Ehrenreich, Appl. Phys. Lett., 61 (24), 2905 (1992). DOI: 10.1063/1.108480

[2] D.L. Smith, C. Mailhiot, J. Appl. Phys., 62 (6), 2545 (1987). DOI: 10.1063/1.339468

[3] M. Razeghi, B.-M. Nguyen, Rep. Prog. Phys., 77, 082401 (2014). DOI: 10.1088/0034-4885/77/8/082401

[4] A. Rogalski, P. Martyniuk, M. Kopytko, Appl. Phys. Rev., 4 (3), 031304 (2017). DOI: 10.1063/1.4999077

[5] J. Wu, Z. Xu, J. Chen, L. He, Infrared Phys. Technol., 92, 18 (2018). DOI: $10.1016 /$ j.infrared.2018.05.004
[6] M.K. Hudait, M. Clavel, P.S. Goley, Y. Xie, J.J. Heremans, Y. Jiang, Z. Jiang, D. Smirnov, G.D. Sanders, C.J. Stanton, Mater. Adv., 1 (5), 1099 (2020). DOI: 10.1039/D0MA00046A

[7] X. Li, J. Cui, Y. Zhao, Q. Wu, Y. Teng, X. Hao, Y. Chen, J. Liu, H. Zhu, Y. Huang, Y. Yao, J. Appl. Phys., 127 (4), 045305 (2020). DOI: $10.1063 / 1.5115269$

[8] G.B. Stringfellow, Organometallic vapor-phase epitaxy, 2nd ed. (Academic Press, London-N.Y., 1999), p. 260. 